\title{
Not even wrong: A philosophical, historical and ultimately a scientific inquiry into the Schmidt Law
}

\author{
Barry F. Madore ${ }^{1}$ and Erika K. Carlson ${ }^{2}$ \\ ${ }^{1}$ The Observatories, Carnegie Institution for Science \\ 813 Santa Barbara Street, Pasadena CA \\ email: barry.f.madore@gmail.com \\ ${ }^{2}$ The Observatories, Carnegie Institution for Science \\ 813 Santa Barbara Street, Pasadena CA \\ email: erikakcarlson@gmail.com
}

\begin{abstract}
We introduce a novel approach to interpreting the well-known spatial correlation of gas densities with on-going star formation. Treated as a closed-loop process involving two physically distinct phases the data can be subdivided into regions that are active and those that are quiescent. The active regions can be distinguished by the presence of high-mass, short-lived, but recently-formed OB stars; the quiescent regions are marked by an absence of these stars and they are considered to be recovering from the last star-formation event and are re-collapsing. The relative (areal) frequencies of those two phases are directly proportional to the relative timescales. For four Local Group galaxies, NGC 6822, the Large \& Small Magellanic Clouds, and M33, the cloud assembly/collapse timescales are all found to be monotonically decreasing power-law functions of density, with as yet to be explained differences.
\end{abstract}

Keywords. Star Formation

\section{Introduction}

For more than half a century the Schmidt Law has been our most popular and widely known empirical parameterization of the rate at which stars are formed from gas. Schmidt (1959) conjectured that the star formation rate (SFR) in the Milky Way could be simply and mono-parametrically expressed as a single power law of the local (volume) gas density: $S F R=\rho_{*} \sim \rho_{\text {gas }}^{n}$. A variety of arguments led Schmidt to suggest that, for stars forming in the neutral hydrogen disk of the Milky Way, the exponent $n$ was in the range of 1-2, with higher values preferred. Madore (1970) gave a dynamical interpretation, which on dimensional grounds led directly to $n=3 / 2$. Correlating the surface density of neutral hydrogen gas with the surface densities of high-mass, young OB stars in the Small Magellanic Cloud, Sanduleak (1969) made the first extragalactic determination, finding $\mathrm{n}=1.84$.

Much impressed by these results, Einasto (1970) was the first to raise the status of these empirical findings from a conjecture to a law. Virtually all subsequent studies adopted this stance and became focused on elucidating the universality of this single (or broken) power-law formulation of the rate of star formation in external galaxies. Most recently Bigiel et al. (2008) surveyed 34 nearby galaxies, and for 18 of them they correlated HI surface densities with multi-wavelength (GALEX and Spitzer) tracers of current star formation. They initially saw an abrupt steepening of the correlation at high (neutral hydrogen) gas densities (their Figure 4). This was quickly explained away when the contribution of molecular hydrogen was added. At high gas densities, neutral 


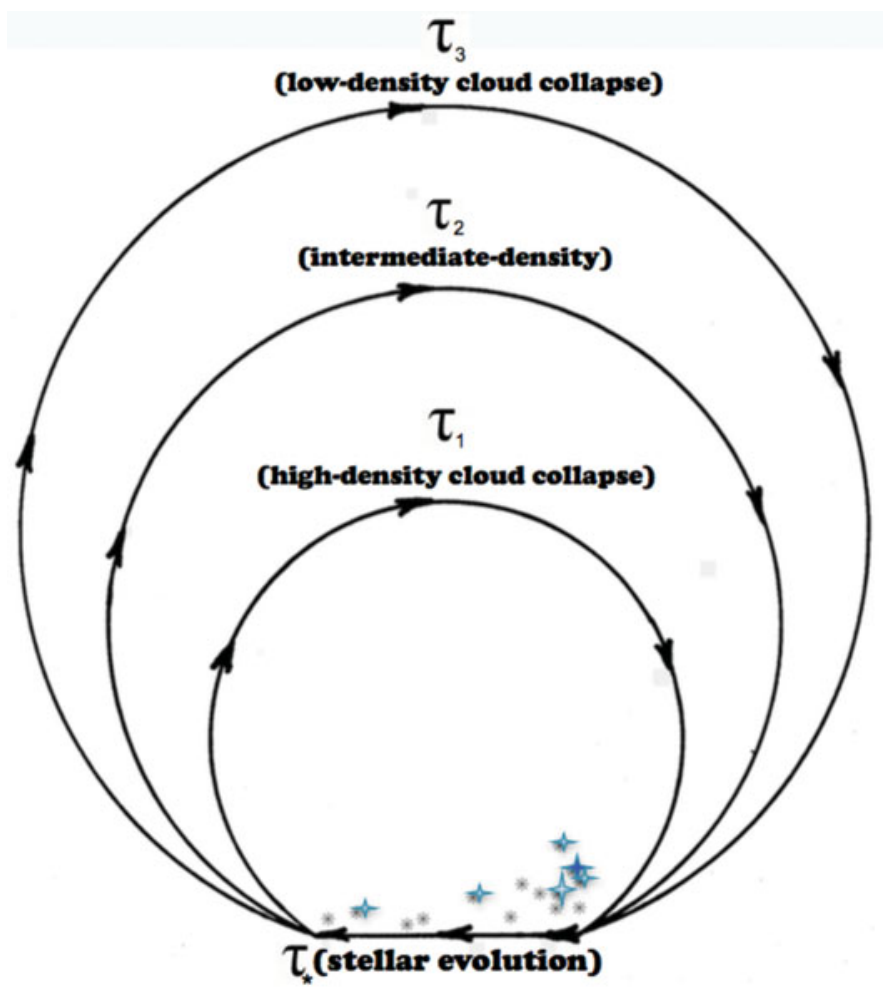

Figure 1. A graphical illustration of the two rate-limiting processes involved in the conversion of gas into stars. The upper loops represent the varying timescales associated with density-dependent cloud assembly and collapse leading up to the star formation event. In this phase no high-mass stars will (yet) be associated with these regions. The single common path (shown at the bottom) connecting all of the upper loops represents the stellar astrophysics path set by the lifetimes of OB stars. The areal frequency of these phases across the face of any given galaxy is directly proportional to amount of time spent in the two (quiescent versus active) phases.

hydrogen is largely depleted as it is converted to its molecular phase, and when this (dominant) contribution was considered, the power law actually flattened out, with $\mathrm{n}=$ 1 prevailing in this highest-density regime. Considerably steeper slopes were still found at low gas densities, but their significance was marginalized in the ensuing discussion as being largely irrelevant to the overall star formation process which was dominantly occurring in giant molecular (sic!) clouds.

However, the broken power law can be interpreted as indicating the existence of two distinct timescales operating in the global cycle of star formation (Madore 2010). The first timescale is the rate-limiting one, involving the low-density neutral hydrogen phase during which clouds assemble, collapse and ultimately begin to form stars. The second timescale is more closely associated (in time) with the immediately-preceding molecular phase, but is ultimately set by stellar evolution timescales of the star-formation tracers (OB stars in particular) that are the final and most visible products of the collapse (see Figure 1). Subsequent work by Elmegreen (2015) has elaborated upon and more fully formalizes this approach to the data. 

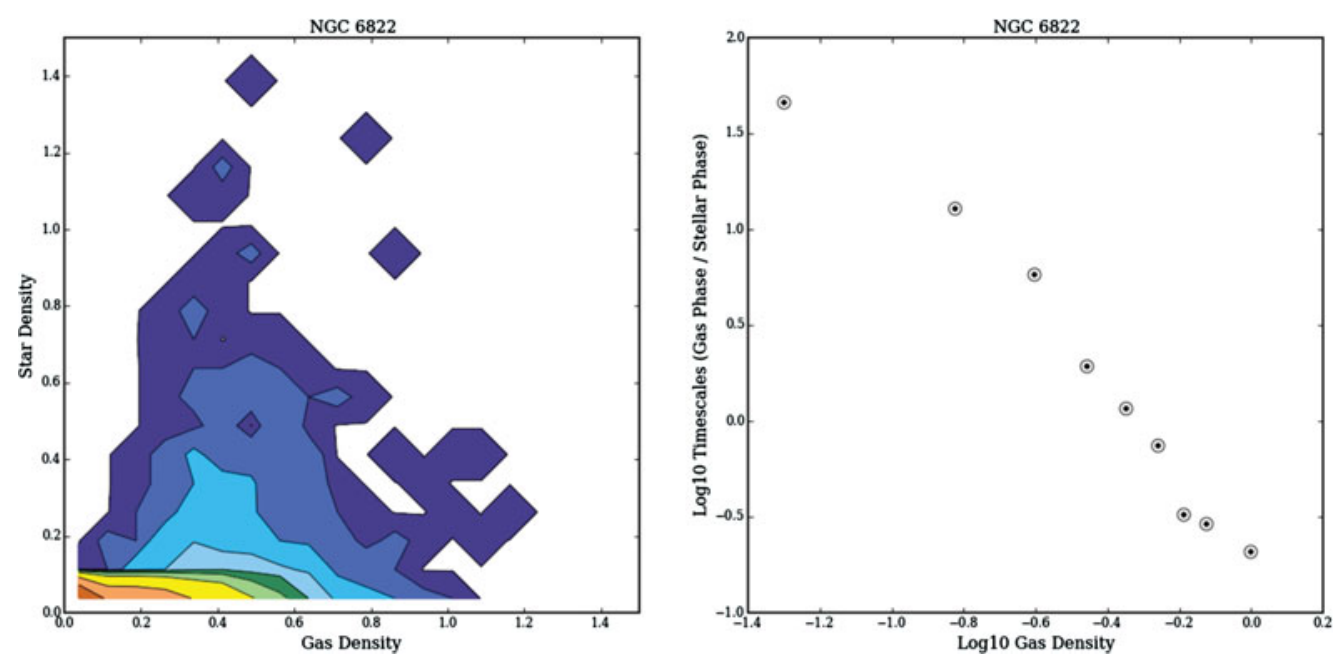

Figure 2. Left Panel - A Star Formation Diagnostic Diagram. Projected OB star densities in NGC 6822, plotted as a function of the locally-projected, neutral hydrogen gas density. Contours represent the frequencies of regions having given stellar surface densities. Note the presence of numerous regions at the base of the plot where there is gas but no stars currently in those cells. Those regions are in the process of collapsing (upper loops in Figure 1). All other regions of the plot currently have recently-formed stars still visible. They are represented by the horizontal base of Figure 1. Right Panel - The monotonic fall of the gas-physics (cloud assembly and collapse) timescale, normalized to the $\mathrm{OB}$ star (astrophysical) lifetimes, as a function of gas density for the galaxy NGC 6822 .

\section{A New Perspective}

Having identified two distinct timescales, associated with two astrophysically decoupled processes, it becomes possible to approach the data in a new way: one that does not invoke a power-law parameterization, and one that does not spatially average over regions in various evolutionary stages, thereby losing information. Rather, this new perspective uses the relative frequencies of regions with and without high-mass stars as being directly proportional to the two rate-limiting timescales. One is a gas-phase timescale, while the other is a stellar-evolution timescale. The left panel of Figure 2 shows the new diagnostic diagram for star formation based on this timescale perspective.

The right panel of Figure 2 illustrates the dependency of these timescale ratios on local gas density. This example is for the Local Group dwarf galaxy NGC 6822. We currently have similar data on three other galaxies (M33, the SMC and LMC) and all four show the same monotonic decrease of cloud assembly-and-collapse timescales with increasing local gas density.

\section{References}

Bigiel, F. 2008, AJ, 136, 2846

Einasto, J. 1972, ApL, 11, 195

Elmegreen, B. G. 2015, ApJ, 814, L30

Madore, B. F. 1977, MNRAS, 178, 1

Madore, B. F. 2010, ApJ, 716, L131

Schmidt, M. 1959, ApJ, 129, 243

Sanduleak, N. 1969, AJ, 74, 47 